\title{
Politische und gesellschaftliche Einstellungen: soziokulturelle Grundllagen von Sellbstorganisation
}

\author{
Luise Burkhardt DIW (SOEP) | Corinna Kausmann DZA | \\ Pascal Siegers GESIS
}

In diesem Kapitel soll auf der Personenebene der Engagierten in Deutschland ein Einblick in die politischen und gesellschaftlichen Einstellungen gegeben werden, die ihrem Engagement zugrunde liegen. Hierzu gehören beispielsweise Wertorientierungen, religiöse Weltanschauungen, demokratische Orientierungen sowie auch die Wahlbeteiligung der Engagierten. Auch Informationen darüber, in welchen gesellschaftlichen und persönlichen Bereichen sich die Engagierten Sorgen machen und wie zufrieden sie allgemein mit ihrem Leben sind, können Aufschluss über die Motivationen der Engagierten geben. Als Datengrundlage dienen der Deutsche Freiwilligensurvey (FWS), die Allgemeine Bevölkerungsumfrage der Sozialwissenschaften (ALLBUS) und das Sozio-oekonomische Panel (SOEP). Den drei Erhebungen liegen unterschiedliche definitorische Konzepte freiwilligen Engagements zugrunde. Während im FWS ein weit gefasster Begriff des Engagements zu finden ist, der auch Engagement, das unabhängig von Organisationen und formalen Ehrenämtern ist, einbezieht, erfolgt im ALLBUS eine Eingrenzung auf Organisationen und Verbände, in denen ein freiwilliges Engagement ausgeführt werden kann. Die Befragten können des Weiteren explizieren, ob es sich dabei um ein Ehrenamt handelt. Im SOEP steht schließlich das klassische Ehrenamt mit einer institutionellen Anbindung an Vereine, Verbände oder Soziale Dienste im Fokus. Die Ergebnisse in diesem Kapitel sind im Hinblick auf diese konzeptionellen Unterschiede zu betrachten. 


\subsection{Wertorientierungen und zivilgesellschaftliches Engagement $^{1}$}

Die Werteforschung hat während der vergangenen 40 Jahre einen fundamentalen Wandel individueller Wertorientierungen in westlichen Gesellschaften beschrieben. Besonders einflussreich war Ronald Ingleharts These, materialistische Werte würden zunehmend durch postmaterialistische Werte abgelöst (Inglehart 1997).

Werte werden in den Sozialwissenschaften als stabile Eigenschaften der Individuen konzipiert. Sie sind abstrakte Prinzipien und dienen als Bewertungsstandards für konkrete Handlungen oder Sachverhalte (Schwartz 1994). Für Inglehart bestimmen die materiellen Bedingungen in der Jugend die Werte der Menschen. Wer in Armut und Unsicherheit aufwächst, wird Werte haben, die das Überleben sichern, insbesondere Konformität mit gesellschaftlichen Normen und Traditionen (Materialismus). Wer hingegen in Wohlstand und Sicherheit aufwächst, wird individuelle Autonomie und Freiheit als Mittel des individuellen Glücks betonen (Postmaterialismus).

In der Diskussion zum Wertewandel wird das Aufkommen postmaterialistischer Werte manchmal als Bedrohung für den gesellschaftlichen Zusammenhalt gedeutet, weil dieser mit einem größeren Egoismus einhergehe. Diese Bedenken konnten in der Literatur weitgehend widerlegt werden (Welzel 2010): postmaterialistische Werte sind nicht nur mit partizipativen (Inglehart und Welzel 2005), sondern auch mit pro-sozialen Orientierungen verbunden (Bekkers 2005).

Dies kann auch anhand der Zusammenhänge zwischen postmateriellen Werten und zivilgesellschaftlichem Engagement in Deutschland gezeigt werden. Die Auswertungen beziehen sich auf Daten aus der Allgemeinen Bevölkerungsumfrage der Sozialwissenschaften (ALLBUS) der Jahre 2008 (GESIS 2015a), 2010 (GESIS 2011) und 2014 (GESIS 2015b).

Zivilgesellschaftliches Engagement wird hier als aktive und passive Mitgliedschaft in Vereinen erfasst. Werte werden anhand des klassischen Inglehart-Index gemessen. BefragungsteilnehmerInnen ordnen dafür vier politische Ziele gemäß ihren Präferenzen zu (Inglehart 1997). Werden zwei postmaterialistische Ziele präferiert, handelt es sich um reine PostmaterialistInnen. Werden zwei materialistische Ziele präferiert, handelt es sich um reine MaterialistInnen. Die Mischtypen werden danach unterschieden, ob das erst gewählte Ziel ein materialistisches oder ein postmaterialistisches Ziel ist.

Die Ergebnisse werden in Abbildung 7.1 für die aktive und passive sowie in Abbildung 7.2 nur für die aktive Vereinsmitgliedschaft dargestellt. Zunächst zeigt sich, dass das zivilgesellschaftliche Engagement über die drei Erhebungszeiträume

1 Dieses Unterkapitel wurde verfasst von Pascal Siegers, GESIS. 
relativ stabil ist. Die Punkte in den Abbildungen 7.1 und 7.2 stellen jeweils den Anteil der Vereinsmitglieder in der Gesamtstichprobe dar. Wie erwartet, ist in allen Fällen der Anteil der Vereinsmitglieder in der Gruppe der Postmaterialisten am höchsten und in der Gruppe der Materialisten am niedrigsten. Die Unterschiede liegen in allen Stichproben bei ca. 20 Prozentpunkten für die Mitgliedschaft in Vereinen und Organisationen (vgl. Abb. 7.1) und bei mindestens 10 Prozentpunkten, was die aktive Mitarbeit betrifft (vgl. Abb. 7.2). Die Mischtypen gleichen in 2010 eher den Materialisten, in 2014 jedoch den Postmaterialisten.

Der Wertewandel hin zu Autonomie und Freiheitswerten unterminiert das zivilgesellschaftliche Engagement nicht. Im Gegenteil, postmaterialistische Wertorientierungen stärken offenbar den partizipativen Anspruch der BürgerInnen nicht nur in der Politik, sondern auch in der Zivilgesellschaft.

Abbildung 7.1 Anteil von Vereinsmitgliedern nach Wertorientierungen (Postmaterialismus) (in Prozent)

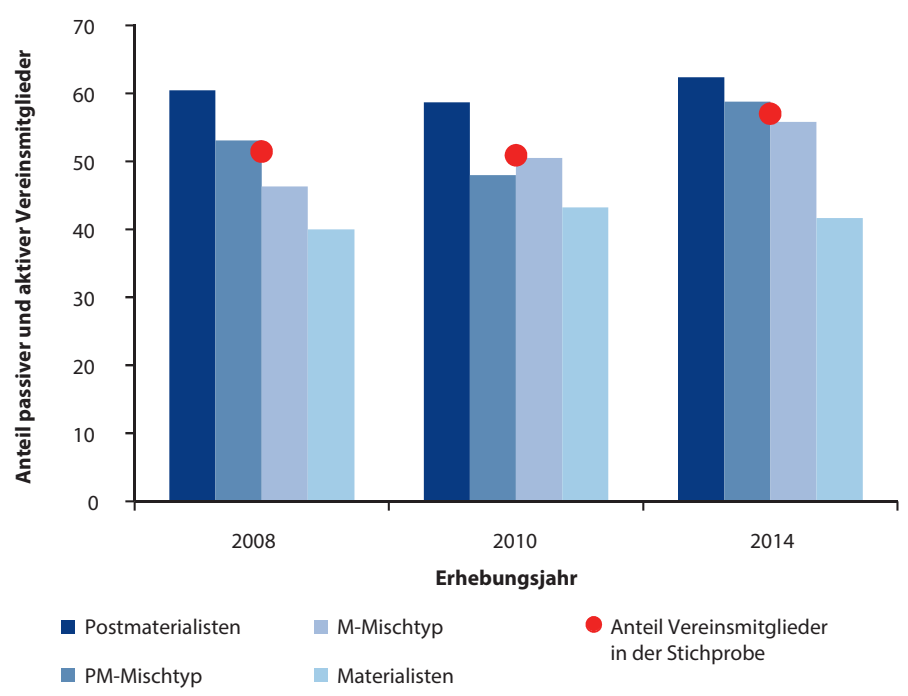

Quelle: Datenbasis ALLBUS 2008, 2010, 2014. Eigene Auswertungen.*

*Zur Korrektur der disproportional geschichteten Stichprobe wurde das Ost-West-Gewicht verwendet. Größe der Stichprobe: 2008: 3 390; 2010: 2 780; 2014: 3 434. Die Frage nach den Vereinsmitgliedschaften umfasste: Sportvereine, Bürgerinitiativen, Wohltätigkeitsvereine, Kulturvereine, Menschenrechtsorganisationen, Naturschutzorganisationen, Gesundheitsvereine, Elternorganisationen, Seniorenvereine, sonstige Hobbyvereine, andere Vereine. 
Abbildung 7.2 Anteil aktiver Vereinsmitglieder nach Wertorientierungen (Postmaterialismus) (in Prozent)

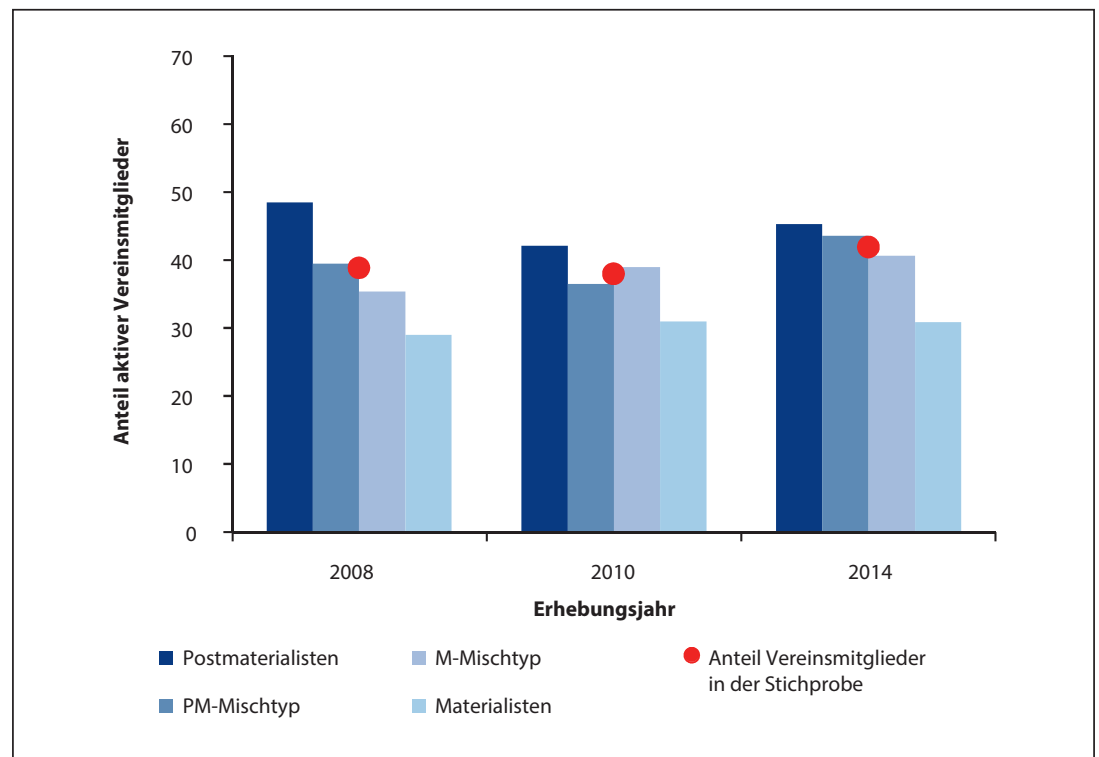

Quelle: Datenbasis ALLBUS 2008, 2010, 2014. Eigene Auswertungen.*

*Zur Korrektur der disproportional geschichteten Stichprobe wurde das Ost-West-Gewicht verwendet. Größe der Stichprobe: 2008: 3 390; 2010: 2 780; 2014: 3 434. Die Frage nach den Vereinsmitgliedschaften umfasste: Sportvereine, Bürgerinitiativen, Wohltätigkeitsvereine, Kulturvereine, Menschenrechtsorganisationen, Naturschutzorganisationen, Gesundheitsvereine, Elternorganisationen, Seniorenvereine, sonstige Hobbyvereine, andere Vereine.

\subsection{Religion und zivilgesellschaftliches Engagement ${ }^{2}$}

In seiner Analyse der amerikanischen Gesellschaft des 19. Jahrhunderts vermutete bereits Alexis de Tocqueville (De la démocratie en Amérique) einen Zusammenhang zwischen der religiösen Konstitution des jungen amerikanischen Bundesstaates und der vitalen Zivilgesellschaft, die er auf seinen Reisen dort vorgefunden hat.

Die empirische Sozialforschung konnte einen Zusammenhang zwischen Religiosität und Engagement empirisch untermauern. In vielen - auch international

2 Dieses Unterkapitel wurde verfasst von Pascal Siegers, GESIS. 
vergleichenden - Auswertungen von Umfragedaten konnte der positive Zusammenhang zwischen der Religiosität - insbesondere über die Häufigkeit des Kirchenbesuchs - und der Bereitschaft zur ehrenamtlichen Mitarbeit in zivilgesellschaftlichen Organisationen nachgewiesen werden (Ruiter und De Graaf 2006).

Die Begründung des beobachteten Zusammenhangs ist in der Literatur noch umstritten. Einerseits könnten religiöse Menschen ihre Kontakte in den Kirchengemeinden dafür nutzen, andere Menschen für die Mitarbeit in ihren Vereinen und Organisationen zu gewinnen. Andererseits betonen die Lehren aller Weltreligionen die Verantwortung ihrer Gläubigen für die Gemeinschaft und insbesondere für ihre schwächeren Mitglieder. Zivilgesellschaftliches Engagement religiöser Individuen kann daher aus dem Glauben heraus motiviert sein (Becker und Dhingra 2001).

Der Zusammenhang zwischen Religiosität und zivilgesellschaftlichem Engagement wird anhand der Daten der Allgemeinen Bevölkerungsumfrage der Sozialwissenschaften (ALLBUS) der Jahre 2008 (GESIS 2015a), 2010 (GESIS 2011) und 2014 (GESIS 2015b) dargestellt.

Zunächst zeigen die Ergebnisse in Abbildung 7.3 und 7.4 große Stabilität im Niveau der aktiven und passiven Vereinsmitgliedschaft über die drei Erhebungen

Abbildung 7.3 Anteil von Vereinsmitgliedern nach Kirchgangshäufigkeit (in Prozent)

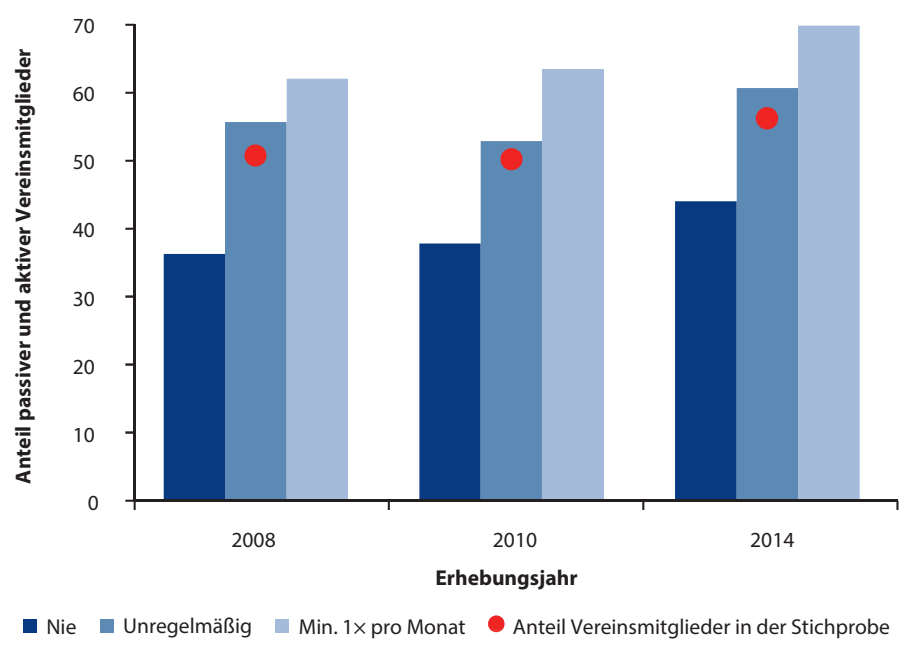

Quelle: Datenbasis ALLBUS 2008, 2010, 2014. Eigene Auswertungen.* 
Abbildung 7.4 Anteil aktiver Vereinsmitglieder nach Kirchgangshäufigkeit (in Prozent)

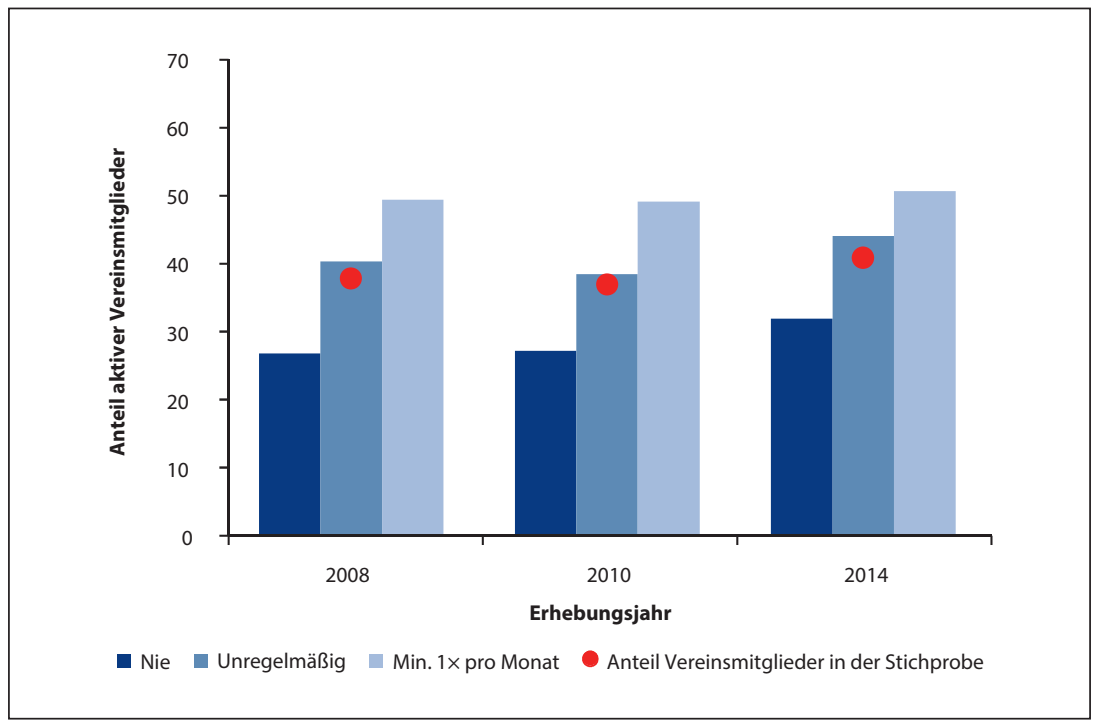

Quelle: Datenbasis ALLBUS 2008, 2010, 2014. Eigene Auswertungen*.

*Zur Korrektur der disproportional geschichteten Stichprobe wurde das Ost-West-Gewicht verwendet. Größe der Stichprobe: 2008: 3 456; 2010: 2 810; 2014: 3 341. Die Frage nach den Vereinsmitgliedschaften umfasste: Sportvereine, Bürgerinitiativen, Wohltätigkeitsvereine, Kulturvereine, Menschenrechtsorganisationen, Naturschutzorganisationen, Gesundheitsvereine, Elternorganisationen, Seniorenvereine, sonstige Hobbyvereine, andere Vereine.

hinweg. Die roten Punkte in der Abbildung stellen jeweils den Anteil der Vereinsmitglieder in der Gesamtstichprobe dar.

Der Zusammenhang zwischen dem Kirchgang und zivilgesellschaftlichem Engagement ist sowohl für die Vereinsmitgliedschaften insgesamt (vgl. Abb. 7.3) als auch für die aktive Mitarbeit in Vereinen (vgl. Abb. 7.4) klar erkennbar. In der Gruppe der regelmäßigen KirchgängerInnen liegt der Anteil der Vereinsmitglieder fast 20 Prozentpunkte höher als in der Gruppe derer, die nie in die Kirche gehen. Mindestens die Hälfte der regelmäßigen KirchgängerInnen ist aktiv in einem Verein oder einer Organisation. Bei den Kirchenfernen ist maximal ein Drittel aktiv engagiert.

Zwei Befunde sind erwähnenswert, die in den Abbildungen jedoch nicht dargestellt sind. Zum einen findet sich der Zusammenhang zwischen Kirchgang und Engagement auch in den neuen Bundesländern, obwohl die individuelle Religiosität in Ostdeutschland deutlich geringer ausgeprägt ist als in Westdeutschland 
(Meulemann 2015). Zum anderen ist kein bedeutsamer Unterschied zwischen Mitgliedern der evangelischen und der römisch-katholischen Kirche in den Daten sichtbar.

Religiosität ist weiterhin eine Ressource für das zivilgesellschaftliche Engagement in Deutschland. Daran hat auch die fortschreitende Säkularisierung nichts geändert. Allerdings verläuft der religiöse Wandel in der Generationenfolge (Crockett und Voas 2006), so dass der Anteil der Bevölkerung, der in den großen christlichen Konfessionen organisiert ist, noch weiter sinken wird. In Zukunft stellt sich daher die Frage, ob auch die neuen Religionsgemeinschaften, die aufgrund von Einwanderung und religiösem Wandel in Deutschland wachsen, zur Vitalität der Zivilgesellschaft beitragen werden (Berghuijs et al. 2013).

\subsection{Demokratische Orientierungen - Politische Partizipation ${ }^{3}$}

Als ein Grundbestandteil der Demokratie ist die politische Partizipation dadurch gekennzeichnet, dass die BürgerInnen politische Entscheidungen beeinflussen oder an ihnen mitwirken (Gabriel 2014). Diese Aktivitäten können sich unterschiedlich darstellen: Man kann unterscheiden nach institutioneller Beteiligung durch Wahlen oder Parteienarbeit und nach nicht-institutionalisierten Formen der Partizipation, wie Demonstrationen oder Unterschriftensammlungen (Weßels 2013). Ebenfalls kann man die politische Partizipation nach vier Typen charakterisieren. Dabei zählen Wahlen als repräsentative Demokratie, Volksentscheide oder Bürgerinitiativen als direkte Demokratie, Demonstrationen sowie Unterschriftenaktionen als demonstrative Demokratie und Beiräte als beratende Demokratie, beispielsweise die Beteiligung an politischen Planungs- und Entscheidungsprozessen (Kersting 2013).

Mit Hilfe des Deutschen Freiwilligensurveys 2014 können vier Varianten der politischen Partizipation dargestellt werden (Simonson und Vogel 2017): politische Ämter, Unterschriftensammlungen oder Online-Petitionen, Demonstrationen und Bürgerinitiativen. Alle Befragten geben für diese vier Formen an, ob sie bisher in ihrem Leben an einer oder mehreren dieser Aktivitäten teilgenommen haben.

Anteilig am häufigsten haben sich die Personen im Alter ab 14 Jahren in Deutschland mit 43,4 Prozent an Unterschriftensammlungen und Online-Petitionen beteiligt. An einer Demonstration haben rund ein Drittel (32,2 Prozent) der Personen und an einer Bürgerinitiative 22,5 Prozent der Personen ab 14 Jahren

3 Dieses Unterkapitel wurde verfasst von Corinna Kausmann, DZA. 
teilgenommen. Deutlich geringer ist der Anteil der Wohnbevölkerung Deutschlands, der bis 2014 einmal im Leben bereits ein politisches Amt übernommen hat (8,7 Prozent).

Deutlich über die Hälfte (58,2 Prozent) der Personen ab 14 Jahren hat in ihrem Leben mindestens an einer der vier betrachteten politischen Partizipationsformen teilgenommen (Abb. 7.5). Während 25,1 Prozent der Menschen an einer der genannten Formen beteiligt waren oder sind, haben 20,1 Prozent zwei Formen beziehungsweise 13,o Prozent drei oder mehr der vorgestellten Partizipationsformen ausgeübt.

Die politische Partizipation unterscheidet sich deutlich zwischen verschiedenen Bildungsgruppen. Während insgesamt 71,5 Prozent der Höhergebildeten sich in ihrem Leben bereits politisch beteiligt haben, sind das bei den Niedriggebildeten 42,o Prozent. Die Anteile der Personen ab 14 Jahren, die bisher eine der vier vorgestellten Formen der politischen Partizipation ausüben oder ausgeübt haben, unterscheiden sich zwischen den Bildungsgruppen nur geringfügig voneinander. Deutliche Unterschiede ergeben sich bei den Anteilen im Vergleich zu denen, die zwei oder mehr Formen der Partizipation angegeben haben. Im Vergleich zu Niedriggebildeten haben mehr als doppelt so viele Personen mit hoher Bildung in

Abbildung 7.5 Anteile derer, die sich in einer oder mehreren politischen Form(en) beteiligt haben, 2014, gesamt, nach Bildung (in Prozent)

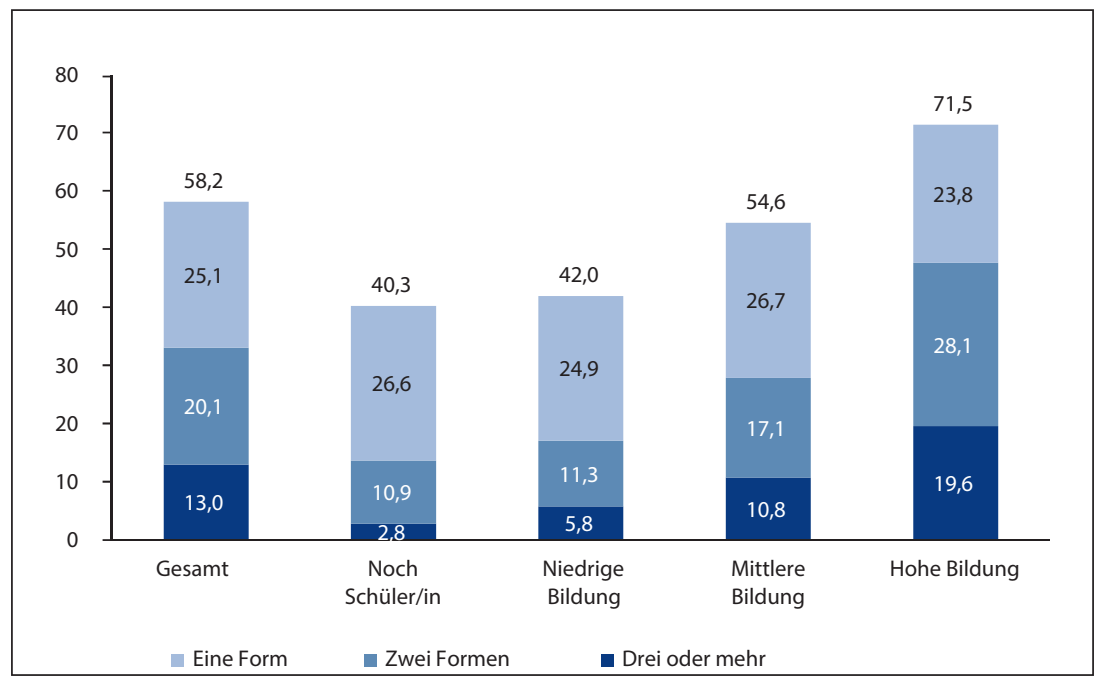

Quelle: FWS 2014, gewichtet, Berechnungen DZA. Basis: Alle Befragten ( $n=28385$ ). 
ihrem Leben zwei Formen der politischen Partizipation ausgeübt. Auch im Vergleich dieser beiden Gruppen in Bezug auf die Ausübung von drei oder mehr Formen sind deutliche Unterschiede zu erkennen (Abb. 7.5). Für die SchülerInnen muss in dieser Betrachtung beachtet werden, dass die geringeren Anteile auch durch das meist noch geringere Lebensalter begründet sind.

Der technische Fortschritt scheint politische Partizipation zu erleichtern. Durch die Nutzung des Internets ist es beispielsweise mehr Menschen möglich, sich zu beteiligen. So zeigt sich, dass Unterschriftensammlungen und Online-Petitionen zu den am häufigsten genannten Formen der Partizipation zählen. Jedoch können auch andere Formen durch Online-Plattformen erleichtert werden oder auch einen Zugang für mehr Menschen schaffen. Dabei sinken nicht nur die Barrieren für die Beteiligung an einer Demonstration oder Bürgerinitiative, sondern auch das Initiieren wird erleichtert. Bei diesen Formen politischer Partizipation werden häufig konkrete Ziele verfolgt, für die mehr oder weniger kurzfristig eine größere Anzahl an UnterstützerInnen mobilisiert werden muss. Ein politisches Amt zu übernehmen ist im Vergleich häufig eine längerfristige Verpflichtung. Bei solchen Ämtern kann es sich um ein Ehrenamt oder um eine bezahlte Beschäftigung handeln.

Die Unterschiede nach Bildungsgruppen werden auch deutlich, wenn man sich die Beteiligung in den einzelnen Formen der politischen Partizipation ansieht (Simonson und Vogel 2017). Zum Beispiel liegen die Anteile derer, die sich schon einmal an einer Unterschriftensammlung oder einer Online-Petition beteiligt haben, bei den Niedriggebildeten bei 26,o Prozent und bei den Hochgebildeten bei 57,8 Prozent. Die politische Partizipation ist nicht nur an öffentliche Gegebenheiten geknüpft, sie wird auch durch den sozialen Status des Einzelnen bedingt. Dabei wirkt sich das Bildungsniveau auf das Interesse an politischen Angelegenheiten und auf das Bewusstsein, Änderungen anstoßen zu können, aus. Es beeinflusst ebenfalls die tatsächlichen Chancen der Mitsprache sowie der Einflussnahme. Durch eine höhere Beteiligung von beispielsweise Höhergebildeten ist es wahrscheinlicher, dass ihre Interessen in politischen Reformen eher berücksichtigt werden und Bedarfe von Personen mit niedriger Bildung durch direktdemokratischen Verfahren unter Umständen nicht gleichberechtigt gehört und einbezogen werden (Geißel 2015). Die politische Partizipation von Personen mit geringeren sozioökonomischen Ressourcen muss gestärkt werden, damit politische Bürgerbeteiligungen nicht zu einer stärkeren sozialen Ungleichheit führen. 


\subsection{Zufriedenheit, Sorgen und zivilgesellschaftliches Engagement ${ }^{4}$}

Die subjektive Lebenszufriedenheit ist ein wichtiges Maß für das Wohlbefinden der Menschen in einer Gesellschaft. Sie wird in der psychologischen und soziologischen Forschung als kognitive Komponente des allgemeinen Wohlbefindens einer Person bezeichnet (Schimmack, Diener und Oichi 2009). Die subjektive Lebenszufriedenheit wird demnach definiert als allgemeine Bewertung der individuellen Lebensqualität einer Person durch diese Person selbst. Dies passiert anhand von eigens zugrunde gelegten Bewertungskriterien (Shin und Johnson 1977) und integriert insbesondere die subjektive Bewertung verschiedener Lebensbereiche auf Basis von vergangenen Erfahrungen, Zukunftserwartungen und des Vergleichs mit anderen Personen (Frey, Luchinger und Stutzer 2004). Das Konzept der subjektiven Lebenszufriedenheit ist demnach zu unterscheiden von den eher affektiven Komponenten persönlichen Wohlbefindens wie beispielsweise situativen negativen und positiven Emotionen, Gefühlen und Stimmungen (Diener 2009).

In diesem Kapitel wird die allgemeine Lebenszufriedenheit von bürgerschaftlich Engagierten im Vergleich zu Nichtengagierten betrachtet. Die Fragen, ob ehrenamtlich Engagierte eine höhere Lebenszufriedenheit aufweisen als Nichtengagierte und inwiefern ein gemeinwohlorientiertes Engagement zu einer höheren Lebenszufriedenheit beiträgt, stehen hierbei im Mittelpunkt.

Als Datenbasis dient das Sozio-oekonomische Panel (SOEP). Die Abfrage der Lebenszufriedenheit erfolgt hier seit 1984 in einem jährlichen Rhythmus auf einer elfstufigen Skala. Freiwilliges Engagement wird im SOEP in einem zweijährlichen Rhythmus erfasst.

In Abbildung 7.6 sind Engagierte im Vergleich zu Nichtengagierten hinsichtlich ihrer durchschnittlichen allgemeinen Lebenszufriedenheit dargestellt.

In Deutschland lebende Personen ab 17 Jahren sind mit einer durchschnittlichen Lebenszufriedenheit von 7 auf einer 11-stufigen Skala allgemein sehr zufrieden. Mit einem durchschnittlichen Wert von 7,3 hat die allgemeine Lebenszufriedenheit der deutschen Bevölkerung im Jahr 2015 ihren bisherigen Höhepunkt erreicht.

Es zeigt sich, dass Personen, die sich freiwillig in Vereinen, Verbänden oder Sozialen Diensten engagieren, durchschnittlich zufriedener sind als Personen, die sich nicht engagieren. Im Jahr 2015 betrug die Differenz dieser beiden Gruppen hinsichtlich ihrer Lebenszufriedenheit 0,3 Skalenpunkte. Diese Tendenz findet sich für alle Beobachtungsjahre und ist mit Hinblick auf das 95-Prozent-Konfidenzniveau signifikant.

4 Dieses Unterkapitel wurde verfasst von Luise Burkhardt, DIW (SOEP). 
Abbildung 7.6 Allgemeine Lebenszufriedenheit von Engagierten und Nicht-Engagierten (1990-2015) (ab 17 Jahren) (in Prozent)

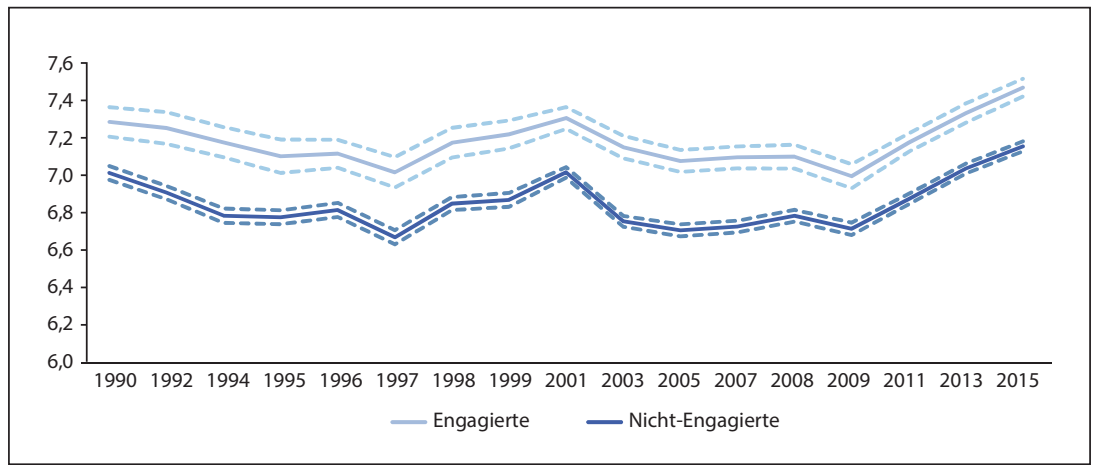

Quelle: SOEP (1990-2015), doi.10.5684/soep.v32, gewichtet, Berechnungen DIW, (Basis: alle Befragten ab 17 Jahren in allen Jahren, bis auf Sample J im Jahr 2011 \& Sample M1 im Jahr 2013).

Abfrage des Ehrenamtes: Welche der folgenden Tätigkeiten üben Sie in Ihrer freien Zeit aus? Geben Sie bitte zu jeder Tätigkeit an, wie oft Sie das machen: jede Woche, jeden Monat, seltener oder nie? - Ehrenamtliche Tätigkeiten in Vereinen, Verbänden oder sozialen Diensten. Abfrage der Lebenszufriedenheit: Wie zufrieden sind Sie gegenwärtig, alles in allem, mit Ihrem Leben? Antworten Sie bitte anhand der folgenden Skala, bei der "o" ganz und gar unzufrieden, "10" ganz und gar zufrieden bedeutet.

Die Richtung des Zusammenhanges von freiwilligem Engagement und allgemeiner Lebenszufriedenheit kann in diesen Analysen nicht festgestellt werden. Die Vermutung liegt aber nahe, dass der Zusammenhang in beide Richtungen besteht. So ist es einerseits möglich, dass sich die regelmäßige Ausübung eines Ehrenamtes durchaus nachhaltig auf die Lebenszufriedenheit auswirkt, da innerhalb des Ehrenamtes beispielsweise soziale Netzwerke aufgebaut und Erfahrungen mit Gleichgesinnten geteilt werden. Gerade auch im Alter, nach dem Eintritt in den Ruhestand, kann ein Ehrenamt eine sinnstiftende Tätigkeit sein und für eine über das Berufsleben hinausgehende Integration in die Gesellschaft sorgen und sich somit positiv auf die Lebenszufriedenheit auswirken (Wahrendorf und Siegrist 2008). Andererseits lässt sich im Ehrenamt auch eine Form der Selbstselektion finden. Das bedeutet, dass Personen, die sich engagieren, oftmals von vornherein über eine höhere Lebenszufriedenheit verfügen als Personen, die sich nicht engagieren (Binder und Freytag 2013).

Neben der allgemeinen Lebenszufriedenheit sind auch die Sorgen, die Personen sich in einer Gesellschaft machen, ein wichtiger und aussagekräftiger Indikator für ihr Wohlbefinden. Unsicherheiten können handlungsleitend wirken, so kann sich die Sorge um den Verlust des Arbeitsplatzes insbesondere bei Frauen 
auf die Bereitschaft zur Familiengründung auswirken (Kreyfeld 2008), Sorgen bezüglich des Umweltschutzes können einen nachhaltigeren Lebensstil hervorrufen und die Sorge vor globalem Terrorismus kann einschränkend auf die Freiheiten der Menschen einer Gesellschaft wirken sowie Ausländerfeindlichkeit schüren. Es kann daher vermutet werden, dass Sorgen auch einen Einfluss auf die Engagementbeteiligung von Personen haben können. Im SOEP werden die Sorgen der Befragten mit rotierenden Themenbereichen jährlich erhoben. In Abbildung 7.7 sind Sorgen dargestellt, die sich eher auf gesellschaftliche Entwicklungen beziehen, in 7.8. solche mit Bezug zur persönlichen Entwicklung. Beide Abbildungen sind absteigend hinsichtlich der größten Sorgen der Engagierten sortiert.

Insgesamt zeigt sich, dass sowohl Engagierte (E) als auch Nichtengagierte (NE) große Sorgen eher mit Bezug auf gesellschaftliche als auf persönliche Entwicklungen äußern. In fast allen im Befragungsjahr 2015 erfragten Sorgen auf gesellschaftlicher Ebene äußern sich die Engagierten etwas besorgter als die Nichtengagierten.

Ein allseits präsentes Thema ist die Sorge um die Erhaltung des Friedens in der Welt. 91 Prozent der Nichtengagierten und rund 93 Prozent der Engagierten äußern diesbezüglich einige und große Sorgen. Weitere Themen sind Umweltschutz, Ausländerfeindlichkeit, der soziale Zusammenhalt, der Klimawandel sowie die allgemeine Wirtschaftsentwicklung. Zwei Entwicklungen auf gesellschaftlicher Ebene stehen die Nichtengagierten besorgter gegenüber als die Engagierten. So sorgen sich rund 84 Prozent (NE) im Vergleich zu rund 81 Prozent (E) um die steigende Kriminalität in Deutschland. Auch hinsichtlich der Zuwanderung von MigrantInnen nach Deutschland zeigen sich Nichtengagierte (ca. 76 Prozent) im Vergleich zu Engagierten (ca. 71 Prozent) besorgter. Hinsichtlich der großen Sorgen in diesen beiden Bereichen unterscheiden sich die beiden Gruppen jeweils sogar um rund 6 Prozentpunkte. Sowohl Nichtengagierte als auch Engagierte sorgen sich außerdem um ihre persönliche Entwicklung. Die Nichtengagierten zeigen sich hier jedoch vermehrt besorgt. 72 Prozent (NE) im Vergleich zu 69 Prozent (E) sorgen sich um die eigene Gesundheit. Dies kann vor allem darin begründet liegen, dass Personen mit einem besseren Gesundheitszustand häufiger im Engagement anzutreffen sind (Müller und Tesch-Römer 2017). Auch im Bereich der eigenen wirtschaftlichen Entwicklung sowie der Arbeitsplatzsicherheit äußern sich die Nichtengagierten etwas häufiger besorgt.

Nicht dargestellt ist in den Abbildungen 7.7 und 7.8 die Antwortkategorie „keine Sorgen“, die die Kategorien „einige Sorgen“ und „große Sorgen“ zu 100 Prozent ergänzt. Bei Hinzunahme dieser Kategorie wird deutlich, dass sich sowohl Engagierte als auch Nichtengagierte vor allem im Bereich der Arbeitsplatzunsicherheit zum großen Teil keine Sorgen machen. So geben 63 Prozent der Engagierten und 58 Prozent der Nichtengagierten an, hier keine Sorgen zu haben. 


\section{Abbildung 7.7 Sorgen um gesellschaftliche Entwicklungen (in Prozent)}

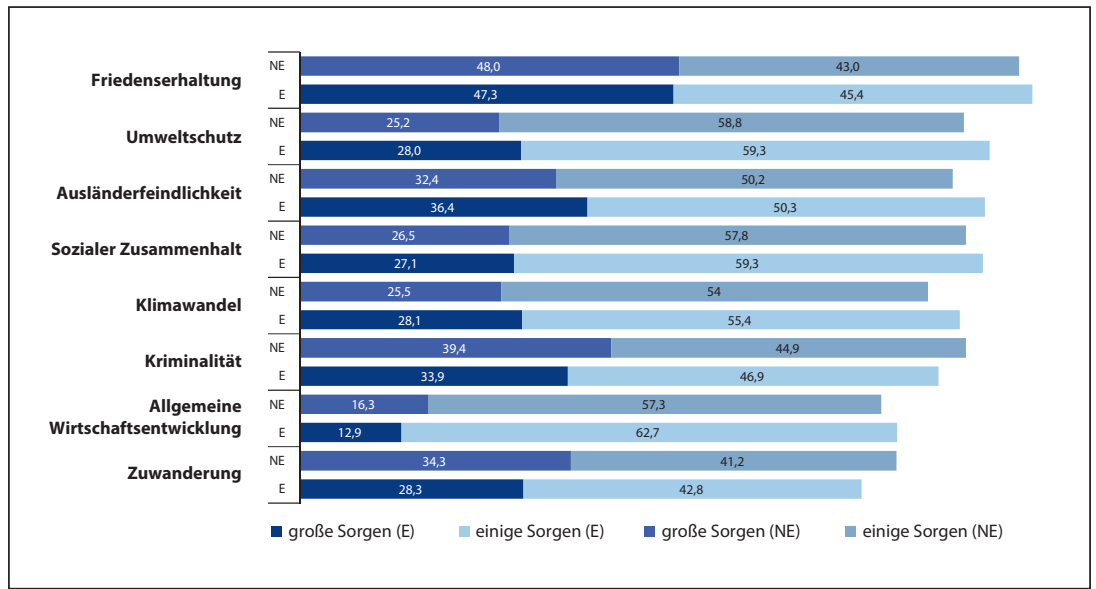

Quelle: SOEP (2015), doi.10.5684/soep.v32., gewichtet, Berechnungen DIW, (Basis: alle Befragten im SOEP 2015 ab 17 Jahren (außer Migrationssample M2, da Frage in Fragebogenversion nicht enthalten) (2015: $n=25394)$. Erhebung der Sorgen: Wie ist es mit den folgenden Gebieten, machen Sie sich da Sorgen?Große Sorgen, einige Sorgen, keine Sorgen. Die dritte Kategorie "keine Sorgen“ ist hier zugunsten der Übersichtlichkeit nicht dargestellt, ergänzt die Balken aber jeweils auf 100 Prozent. (NE) = Nichtengagierte, $(\mathrm{E})=$ Engagierte.

Abbildung 7.8 Sorgen um persönliche Entwicklungen (in Prozent)

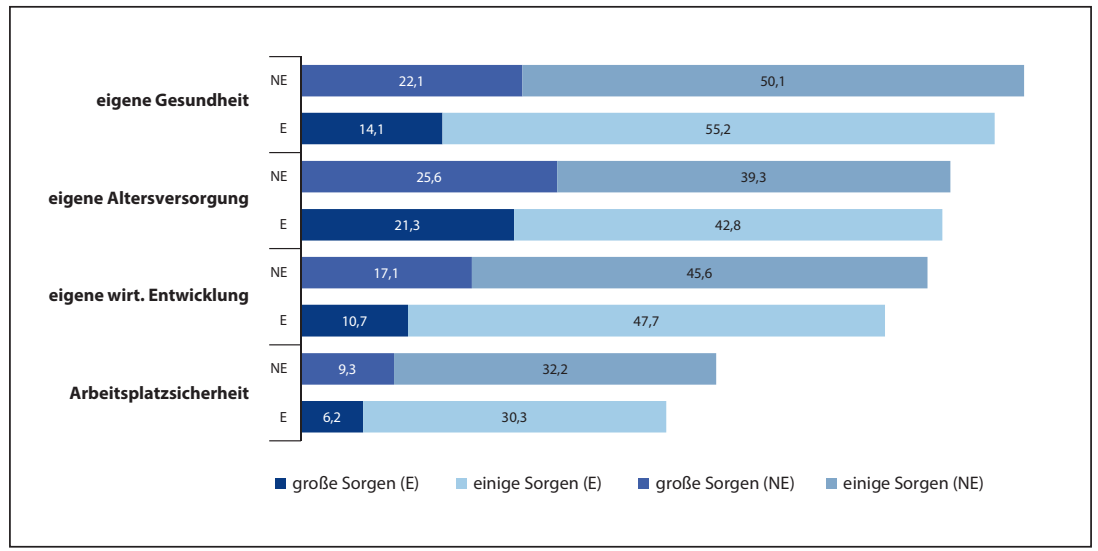

Quelle: SOEP (2015), doi.10.5684/soep.v32., gewichtet, Berechnungen DIW, (Basis: alle Befragten im SOEP 2015 ab 17 Jahren (außer Migrationssample M2, da Frage in Fragebogenversion nicht enthalten) (2015: $\mathrm{n}=25394)(\mathrm{NE})=$ Nichtengagierte $(\mathrm{E})=$ Engagierte. 
Insgesamt lässt sich sagen, dass sich die Engagierten und die Nichtengagierten hinsichtlich ihrer Sorgenstrukturen geringfügig voneinander unterscheiden. Insbesondere die Entwicklung der Sorgen im Bereich Kriminalität und Zuwanderung nach Deutschland ist für weitere Analysen, besonders im Hinblick auf den Zustrom von Geflüchteten, den Deutschland seit 2015 verzeichnet, von Interesse.

\subsection{Literatur}

Becker, P. E., \& Dhingra, P. H. (2001). Religious involvement and volunteering: Implications for civil society. Sociology of Religion, 62(3), 315-335, doi:Doi 10.23 $07 / 3712353$.

Bekkers, R. (2005). Participation in voluntary associations: Relations with resources, personality, and political values. Political Psychology, 26(3), 439-454, doi:DOI 10.1111/j.1467-9221.2005.00425.x.

Berghuijs, J., Pieper, J., \& Bakker, C. (2013). New Spirituality and Social Engagement. Journal for the Scientific Study of Religion, 52(4), 775-792, doi:10.1111/jssr.12062.

Binder, M., \& Freytag, A. (2013). Volunteering, Subjective Well-Being and Public Policy. Journal of Economic Psychology 34. (S. 97-119).

Crockett, A., \& Voas, D. (2006). Generations of Decline: Religious Change in 2othCentury Britain. Journal for the Scientific Study of Religion, 45(4), 567-584.

Diener, E. (2009). Assessing Subjective Well-Being: Progress and Opportunities. In E. Diener, W. Glatzer, T. Moum, M. A. G. Sprangers, J. Vogel \& R. Veenhoven (Hrsg.), Assessing well-being. The collected works of Ed Diener (S. 25-65). Springer Science+Business Media B. V.

Frey, B. S., Luechinger, S., \& Stutzer, A. (2004). Valuing Public Goods: The Life Satisfaction Approach, CESifo Working Paper, No. 1158.

Gabriel, O. (2014). Bürgerbeteiligung in Deutschland. In K. Pohl \& P. Massing (Hrsg.) Mehr Partizipation - mehr Demokratie? (S. 27-45). Schwalbach/Taunus: Wochenschau Verlag.

Geißel, B. (2015). Mitwirkung für alle? Thesen zur politischen Ungleichheit. In L. Harles \& D. Lange (Hrsg.), Zeitalter der Partizipation. Paradigmenwechsel in Politik und politischer Bildung? (S. 37-48). Schwalbach/Taunus: Wochenschau Verlag.

GESIS - Leibniz-Institut für Sozialwissenschaften (2015a). Allgemeine Bevölkerungsumfrage der Sozialwissenschaften ALLBUS 2008. GESIS Datenarchiv, Köln. ZA460o Datenfile Version 2.1.o, doi:10.4232/1.12345. Zugegriffen: 21. November 2017.

GESIS - Leibniz-Institut für Sozialwissenschaften (2015b). Allgemeine Bevölkerungsumfrage der Sozialwissenschaften ALLBUS 2014. GESIS Datenarchiv, Köln. ZA5240 Datenfile Version 2.1.0, doi:10.4232/1.12288. Zugegriffen: 21. November 2017 . 
GESIS - Leibniz-Institut für Sozialwissenschaften (2011). Allgemeine Bevölkerungsumfrage der Sozialwissenschaften ALLBUS 2010. GESIS Datenarchiv, Köln. ZA4610 Datenfile Version 1.1.o, doi:10.4232/1.10760. Zugegriffen: 21. November 2017 .

Inglehart, R. (1997). Modernziation and postmodernization: cultural, economic, and political change in 43 societies. Princeton: Princeton University Press.

Inglehart, R., \& Welzel, C. (2005). Modernization, Cultural Change, and Democracy. Cambridge/New York: Cambridge University Press.

Kersting, N. (2013). Wutbürger und andere soziale Bewegungen. Der Bürger im Staat, 65(3), S. 155-165.

Kreyenfeld, M. (2008). Ökonomische Unsicherheit und der Aufschub der Familiengründung. In M. Szydlik (Hrsg.) Flexibilisierung (S. 232-254). Wiesbaden: VS Verlag für Sozialwissenschaften.

Kühnemund, H. (2006). Methodenkritische Anmerkungen zur Empirie ehrenamtlichen Engagements. In K. R. Schroeter \& P. Zängl (Hrsg.), Altern und bürgerschaftliches Engagement. Aspekte der Vergemeinschaftung und Vergesellschaftung in der Lebensphase Alter (S. 111-134). Wiesbaden: VS Verlag für Sozialwissenschaften.

Meulemann, H. (2015). Nach der Säkularisierung: Religiosität in Deutschland 1980-2012.

Müller, D., \& Tesch-Römer, C. (2017). Krankheitsbedingte Alltagseinschränkungen, subjektive Gesundheit, Lebenszufriedenheit und freiwilliges Engagement. In J. Simonson, C. Vogel \& C. Tesch-Römer (Hrsg.), Freiwilliges Engagement in Deutschland - Der Deutsche Freiwilligensurvey 2014 (S. 495-482). Wiesbaden: Springer VS.

Poutvaara, P., \& Steinhardt, M.F. (2015). Bitterness in life and attitudes towards immigration (October 2015). SOEPpaper No. 80o. Verfügbar unter SSRN: https:// ssrn.com/abstract=2698613. Zugegriffen: 21 . November 2017.

Ruiter, S., \& De Graaf, N. D. (2006). National Context, Religiosity, and Volunteering: Results from 53 Countries. American Sociological Review, 71(2), 191-210.

Schimmack, U., Diener, E., \& Shigehiro, O. (2009). Life-Satisfaction Is a Momentary Judgment and a Stable Personality Characteristic: The Use of Chronically Accessible and Stable Sources. In E. Diener, W. Glatzer, T. Moum, M. A. G. Sprangers, J. Vogel \& R. Veenhoven (Hrsg.), Assessing well-being. The collected works of Ed Diener (S. 181-211). Springer Science+Business Media B. V.

Schupp, J. (2009). 25 Jahre Sozio-oekonomisches Panel - Ein Infrastrukturprojekt der empirischen Sozial- und Wirtschaftsforschung in Deutschland. Zeitschrift für Soziologie 38(5), 350-357.

Schwartz, S. H. (1994). Are There Universal Aspects in the Structure and Contents of Human Values? Journal of Social Issues, 50(4), 19-45.

Shin, D. C., \& Johnson, D. M. (1978). Avowed happiness as an overall assessment of the quality of life. Social Indicators Research. 5(1-4). S. 475-492.

Simonson, J., \& Vogel, C. (2017). Politische Partizipation: Unterschriftenaktionen, Demonstrationen, Bürgerinitiativen und politische Ämter. In J. Simonson, C. Vogel \& C. Tesch-Römer (Hrsg.), Freiwilliges Engagement in Deutschland - Der Deutsche Freiwilligensurvey 2014 (S. 199-216). Wiesbaden: Springer VS. 
Weßels, B. (2013). Politische Integration und politisches Engagement. In Statistisches Bundesamt \& Wissenschaftszentrum Berlin für Sozialforschung (Hrsg.), $D a$ tenreport 2013. Ein Sozialbericht für die Bundesrepublik Deutschland (S. 363376). Bonn: Bundeszentrale für politische Bildung.

Wahrendorf, M., \& Siegrist, J. (2008). Soziale Produktivität und Wohlbefinden im höheren Lebensalter. In M. Ehrlinghagen \& K. Hank (Hrsg.), Produktives Altern und informelle Arbeit in modernen Gesellschaften. Theoretische Perspektiven und empirische Befunde (S. 51-74.).Wiesbaden: VS Verlag für Sozialwissenschaften. Welzel, C. (2010). How Selfish Are Self-Expression Values? A Civicness Test. Journal of Cross-Cultural Psychology, 41(2), 152-174.

Open Access Dieses Kapitel wird unter der Creative Commons Namensnennung 4.0 International Lizenz (http://creativecommons.org/licenses/by/4.0/deed.de) veröffentlicht, welche die Nutzung, Vervielfältigung, Bearbeitung, Verbreitung und Wiedergabe in jeglichem Medium und Format erlaubt, sofern Sie den/die ursprünglichen Autor(en) und die Quelle ordnungsgemäß nennen, einen Link zur Creative Commons Lizenz beifügen und angeben, ob Änderungen vorgenommen wurden.

Die in diesem Kapitel enthaltenen Bilder und sonstiges Drittmaterial unterliegen ebenfalls der genannten Creative Commons Lizenz, sofern sich aus der Abbildungslegende nichts anderes ergibt. Sofern das betreffende Material nicht unter der genannten Creative Commons Lizenz steht und die betreffende Handlung nicht nach gesetzlichen Vorschriften erlaubt ist, ist für die oben aufgeführten Weiterverwendungen des Materials die Einwilligung des jeweiligen Rechteinhabers einzuholen.

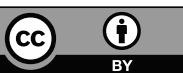

